\title{
Efficiency and Consistency for Locating Multiple Public Facilities
}

\author{
Biung-Ghi Ju*
}

November 4, 2005

\begin{abstract}
In the problem of locating multiple public facilities studied by Barberà and Beviá (2002, 2005), we offer simple necessary and sufficient conditions for efficiency, decentralizability of efficient decisions in a game of community division and local public goods provision, and a constructive algorithm for efficient and consistent decisions.
\end{abstract}

Keywords: Efficiency; Consistency; Self-selection consistency; No-envy; Local stability; Diversity; Strong Nash equilibrium; Community division; Location

${ }^{*}$ Department of Economics, The University of Kansas, 1300 Sunnyside Avenue, Lawrence, KS 66045, USA. E-mail: bgju@ku.edu. Tel.:+1-785-864-2860. Fax.:+1-785-864-5270. Acknowledgement will be added later. 


\section{Introduction}

Barberà and Beviá $(2002,2005)$ introduce a notion of consistency between a global decision governing community-wise consumptions and local decisions by individual communities. They consider a simple model of community division and location of (local) public facilities. ${ }^{1}$ A location is chosen from a real line. Members of a community have single-peaked preferences over the line and the size of the community does not affect members' welfare (no congestion effect). Given a set of agents, their preferences, and a fixed number of communities to form, a social choice function determines a community division and locations of public facilities for individual communities. The function is efficient if its decision does not allow any further welfare enhancement (making all agents weakly better off and at least one better off). It is consistent if any local component (a community and the location of public facility for this community) of its global decision coincides with the local decision made by applying the function for this community only.

We offer simple necessary and sufficient conditions for efficiency and decentralizability of efficient decisions à la the first and the second welfare theorems. Next, building on a constructive argument used by Barberà and Beviá (2002) in their proof of Proposition 1, we define an algorithm that can be used to design efficient and consistent collective decision functions.

Our conditions for efficiency are composed of the following four axioms: no-envy (everyone weakly prefers the location in his own community to the location in any other community), local stability (there is no community where some members voluntarily move out and all remaining members agree on relocating their facility), and two diversity conditions, location diversity and community diversity.

The decentralizability result is obtained, using strong Nash equilibrium, in a game of community division and local public goods provision, similar to the game considered by Konishi, Le Breton, and Weber (1997a, 1997b). ${ }^{2}$ Our conditions for efficiency play a critical role in proving this decentralizability result and existence of efficient and consistent collective decision functions.

The rest of the paper is organized as follows. We define our model and basic concepts in Section 2. In Section 3, we offer necessary and sufficient conditions for

\footnotetext{
${ }^{1}$ A simpler model of locating two public facilities is studied by Miyagawa (2001) and Ehlers (2002, 2003).

${ }^{2}$ As in Konishi, Le Breton, and Weber (1997a, 1997b), players are partitioned into communities each of which consists of players with the same strategy choice. However, our model differs crucially from theirs in that there is no externality or rivalry and the number of communities that can form is exogenous. Considering this game, we study the relationship between efficient decisions and strong Nash equilibria, while the main objective of Konishi, Le Breton, and Weber (1997a, 1997b) is to study conditions for existence of strong Nash equilibria in a more general environment than ours.
} 
efficiency (Section 3.1), and decentralizability of efficient decisions (Section 3.2). In Section 4, we show existence of efficient and consistent functions. Proofs of some results are provided in the appendix.

\section{The Model and Basic Concepts}

We consider the following collective decision problem. There is a set of agents. These agents have to be divided into a fixed number of subsets, called communities, and for each of these communities, a location for a local public facility has to be decided. A location is represented by a real number and the real line $\mathbb{R}$ is the set of possible locations. We consider these problems in a variable population environment and use natural numbers in $\mathbb{N}$ to label agents. Let $P \subseteq \mathbb{N}$ be the set of potential agents. A population is a finite subset of $P$. Let $\mathscr{N}$ be the set of all populations. Given a population $N \in \mathscr{N}$ and a number of communities $\bar{k} \in \mathbb{N}$ with $\bar{k} \leq|N|$, let $\Pi(N, \bar{k})$ be the set of profiles of $\bar{k}$-subsets, $\left(C_{1}, \cdots, C_{\bar{k}}\right)$, which constitute a partition of $N$. An $N / \bar{k}$-decision is a list of $\bar{k}$-communities, $\left(C_{1}, \cdots, C_{\bar{k}}\right) \in \Pi(N, \bar{k})$, and the locations of public facilities for these communities, $\left(x_{1}, \cdots, x_{\bar{k}}\right) \in(\mathbb{R} \cup\{v\})^{\bar{k}}$, where $v$ means "no location (or no public facility)". Let $Z(N, \bar{k})$ be the set of $N / \bar{k}$-decisions. For each $N / \bar{k}$-decision $z$ and each $k=1, \cdots, \bar{k}$, let $z_{k} \equiv\left(C_{k}, x_{k}\right)$ be the pair of community- $k$ and the location for community- $k$. For simplicity, each $N / \bar{k}$-decision $z \in Z(N, \bar{k})$ is labeled in such a way that $x_{1} \leq x_{2} \leq \cdots \leq x_{\bar{k}}$. Let $Z(\bar{k}) \equiv \cup_{N \in \mathscr{N}} Z(N, \bar{k})$ and $Z \equiv \cup_{\bar{k} \in \mathbb{N}} Z(\bar{k})$. In what follows, we use indices $h, i, j$ for agents and indices $k, l, m$ for communities. Components of $z$ and $z^{\prime}$ are denoted by $z_{k}=\left(C_{k}, x_{k}\right)$ and $z_{k}^{\prime}=\left(C_{k}^{\prime}, x_{k}^{\prime}\right)$ respectively for all $k=1, \ldots, \bar{k}$.

Each agent has a preference relation over $Z$. We assume that each agent cares about only the location of the public facility for the community he belongs to: that is, for all $z, z^{\prime} \in Z$, if $i \in C_{k}, i \in C_{l}^{\prime}$, and $x_{k}=x_{l}^{\prime}$, then $z$ and $z^{\prime}$ are indifferent for $i$. Thus in our model, agents have no preference over community members or community sizes. Under this assumption, preferences over $Z$ can be described as preferences over the set of locations $\mathbb{R} \cup\{v\}$. The generic notation for a preference relation for agent $i$ is $R_{i}$. The strict and the indifferent counterparts of $R_{i}$ are denoted by $P_{i}$ and $I_{i}$ respectively. For each $x, x^{\prime} \in \mathbb{R} \cup\{v\}$, we write $x P_{i} x^{\prime}$ when agent $i$ prefers $x$ to $x^{\prime} ; x I_{i} x^{\prime}$ when the two are indifferent; $x R_{i} x^{\prime}$ when $x P_{i} x^{\prime}$ or $x I_{i} x^{\prime}$.

Furthermore, we assume that each agent $i$ has a single-peaked preference relation $R_{i}$ over $\mathbb{R} \cup\{v\}$, that is, there is the peak location $p\left(R_{i}\right) \in \mathbb{R}$ such that for each $x, x^{\prime} \in \mathbb{R}$, if $x^{\prime}<x \leq p\left(R_{i}\right)$ or $p\left(R_{i}\right) \leq x<x^{\prime}, x P_{i} x^{\prime}$, and that for each $x \in \mathbb{R}, x P_{i} v$ (thus having a local public facility wherever it is located is better than not having one). Let $\mathscr{R}$ be the set of single-peaked preferences. For each $N \in \mathscr{N}$, let $\mathscr{R}^{N}$ be the set of profiles of single-peaked preferences of agents in $N$. 
For each population $N \in \mathscr{N}$, a problem for $N$ is characterized by a list of preferences of agents in $N, R \in \mathscr{R}^{N}$, and a number of communities to form, $\bar{k} \in \mathbb{N}$ with $\bar{k} \leq|N|$. Let $\mathscr{D}^{N} \subseteq \mathscr{R}^{N} \times \mathbb{N}$ be the set of all these problems for $N$. Let $\mathscr{D} \equiv \cup_{N \in \mathscr{N}} \mathscr{D}^{N}$. A social choice function $\varphi: \mathscr{D} \rightarrow Z$ maps each problem $(R, \bar{k}) \in \mathscr{D}$ with population $N \in$ $\mathscr{N}$ into an $N / \bar{k}$-decision, that is, $\varphi(R, \bar{k}) \in Z(N, \bar{k})$. Each social choice function $\varphi$ can be described by two component functions $\varphi^{C}: \mathscr{D} \rightarrow \cup_{\bar{k} \in \mathbb{N}, N \in \mathscr{N}} \Pi(N, \bar{k})$ and $\varphi^{L}: \mathscr{D} \rightarrow \cup_{\bar{k} \in \mathbb{N}} \mathbb{R}^{\bar{k}}$ such that for each $N \in \mathscr{N}$ and each $(R, \bar{k}) \in \mathscr{R}^{N} \times \mathbb{N}, \varphi^{C}(R, \bar{k})$ is the list of $\bar{k}$-communities in $\varphi(R, \bar{k})$ and $\varphi^{L}(R, \bar{k})$ is the list of $\bar{k}$-locations in $\varphi(R, \bar{k})$.

We now state two main axioms of social choice functions, which are crucial in this paper. Given $N \in \mathscr{N}, \bar{k} \in \mathbb{N}$, and $R \in \mathscr{R}^{N}$, an $N / \bar{k}$-decision $z \equiv\left(C_{k}, x_{k}\right)_{k=1}^{\bar{k}}$ is a Pareto improvement of another $N / \bar{k}$-decision $z^{\prime} \equiv\left(C_{k}^{\prime}, x_{k}^{\prime}\right)_{k=1}^{\bar{k}}$ at $R$, if all agents weakly prefer $z$ to $z^{\prime}$ and at least one agent prefers $z$ to $z^{\prime}$ : that is, for each $i \in N$ and each $k, l=1, \cdots, \bar{k}$ with $i \in C_{k} \cap C_{l}^{\prime}, x_{k} R_{i} x_{l}$, and for some $j \in N$ and $m, n=1, \cdots, \bar{k}$ with $j \in C_{m} \cap C_{n}^{\prime}, x_{m} P_{i} x_{n}$. An $N / \bar{k}$-decision is efficient if there is no Pareto improvement of the decision.

Efficiency. For each $N \in \mathscr{N}$, each $R \in \mathscr{R}^{N}$, and each $\bar{k} \in \mathbb{N}$, there is no Pareto improvement of $\varphi(R, \bar{k})$ at $R$.

The next axiom introduced by Barberà and Beviá (2002) is a basic consistency requirement between (global) decisions for the whole population and local decisions for individual communities. Suppose that after an $N / \bar{k}$-decision is made, each community can reassess its own location. The next axiom says that the original $N / \bar{k}$-decision should remain intact after the community-wise reassessment.

Consistency. For each $N \in \mathscr{N}$, each $(R, \bar{k}) \in \mathscr{R}^{N} \times \mathbb{N}$, and each $k \in\{1, \cdots, \bar{k}\}$, $\varphi_{k}(R, \bar{k})=\varphi\left(R_{C_{k}}, 1\right)$, where $C_{k}$ denotes community- $k$ in $\varphi(R, \bar{k})$.

A social choice function satisfies self-selection consistency (Barberà and Beviá 2002) if it satisfies both efficiency and consistency.

\section{Efficiency}

\subsection{Conditions for Efficiency}

In this section, we identify necessary and sufficient conditions for efficiency.

As pointed out by Barberà and Beviá (2002), the following are two necessary conditions for efficiency. To define them, we use the following notation. For each $N \in \mathscr{N}$, 
each $R \in \mathscr{R}^{N}$, and each $S \subseteq N$, let $R_{S} \equiv\left(R_{i}\right)_{i \in S}$. And let $p_{*}\left(R_{S}\right) \equiv \min \left\{p\left(R_{i}\right): i \in S\right\} ; p^{*}\left(R_{S}\right) \equiv \max \left\{p\left(R_{i}\right): i \in S\right\} ; P\left(R_{S}\right) \equiv\left[p_{*}\left(R_{S}\right), p^{*}\left(R_{S}\right)\right]$.

We call $P\left(R_{S}\right)$ the local Pareto set for community $S$ with $R_{S}$.

For each $N \in \mathscr{N}$ and each $(R, \bar{k}) \in \mathscr{D}^{N}$, an $N / \bar{k}$-decision $z \equiv\left(C_{k}, x_{k}\right)_{k=1}^{\bar{k}}$ satisfies no-envy if for each $k=1, \cdots, \bar{k}$, each $i \in C_{k}$, and each $l=1, \cdots, \bar{k}, x_{k} R_{i} x_{l}$. It satisfies local efficiency if for each $k \in\{1, \cdots, \bar{k}\}, x_{k} \in P\left(R_{C_{k}}\right)$. The two conditions are not sufficient for efficiency. This is shown by the three examples below. These examples will also provide us some insight into what additional conditions are needed for efficiency.

Example 1. Let $N \equiv\{1,2,3\}$ and $\bar{k} \equiv 2$. Let $R \in \mathscr{R}^{N}$ be a profile such that $p\left(R_{1}\right)=1$, $p\left(R_{2}\right)=2, p\left(R_{3}\right)=3$, and $1.5 I_{2} 3$. Consider the $N / \bar{k}$-decision $z \equiv\left(\left(C_{1}, x_{1}\right),\left(C_{2}, x_{2}\right)\right)$ defined as follows: $C_{1} \equiv\{1,2\}, C_{2} \equiv\{3\}, x_{1} \equiv 1.5$, and $x_{2} \equiv 3$. Then if we move agent 2 into community- 2 and change the location for community- 1 to $x_{1}^{\prime} \equiv 1$, agent 1 is better off and agents 2 and 3 are indifferent. Therefore, $z$ is not efficient.

The Pareto improvement of $z$ in the example is possible because for a community (community 2 in the example), the location is not between the peaks of members for whom it is the preferred location among all locations in $z$. Such Pareto improvement does not exist if $z$ satisfies the next condition.

For each $N / \bar{k}$-decision $z \equiv\left(C_{k}, x_{k}\right)_{k=1}^{\bar{k}}$, each $R \in \mathscr{R}^{N}$, and each $k \in\{1, \cdots, \bar{k}\}$, let

$$
C_{k}^{0}(R, z) \equiv\left\{i \in C_{k}: \text { for each } l=1, \cdots, \bar{k}, \text { if } x_{l} \neq x_{k}, x_{k} P_{i} x_{l}\right\}
$$

be the set of members of community- $k$ who prefer the location for this community to any different location for other communities. Note that for each $i \in C_{k} \backslash C_{k}^{0}(R, z)$, $x_{k} \neq p\left(R_{i}\right)$ and there is $l \neq k$ such that $x_{l} \neq x_{k}$ and $x_{l} R_{i} x_{k}$. Thus if $x_{k} \notin P\left(R_{C_{k}^{0}(R, z)}\right)$, then moving all agents in $C_{k} \backslash C_{k}^{0}(R, z)$ to other communities they weakly prefer and changing the location for community- $k$ to the closest location in $P\left(R_{C_{k}^{0}(R, z)}\right)$ improves welfare of all remaining members in community- $k$ without hurting anyone else. The next axiom guarantees that there be no such Pareto improvement. An $N / \bar{k}$-decision $z$ satisfies local stability if for each $k=1, \cdots, \bar{k}, x_{k} \in P\left(R_{C_{k}^{0}(R, z)}\right)$. Clearly, this axiom implies local efficiency but the converse does not hold as shown by Example 1. The next example shows that the combination of no-envy and local stability is not sufficient for efficiency.

Example 2. Let $N \equiv\{1,2,3,4\}$ and $\bar{k}=2$. Let $R \in \mathscr{R}^{N}$ be a profile such that $p\left(R_{1}\right)=$ 1, $p\left(R_{2}\right)=p\left(R_{3}\right)=2$, and $p\left(R_{4}\right)=3$. Let $z \equiv\left(\left(C_{1}, x_{1}\right),\left(C_{2}, x_{2}\right)\right)$ be the $N / \bar{k}$-decision defined as follows: $C_{1} \equiv\{1,2\}, x_{1} \equiv 2, C_{2} \equiv\{3,4\}$, and $x_{2} \equiv 2$. Note that $z$ satis- 
fies both no-envy and local stability. However, $z$ violates efficiency because there is a Pareto improvement $z^{\prime} \equiv\left(\left(C_{1}^{\prime}, x_{1}^{\prime}\right),\left(C_{2}^{\prime}, x_{2}^{\prime}\right)\right)$ defined as follows: $C_{1}^{\prime} \equiv\{1\}, x_{1}^{\prime} \equiv 1$, $C_{2}^{\prime} \equiv\{2,3,4\}$, and $x_{2}^{\prime} \equiv 2$.

The $N / \bar{k}$-decision $z$ in Example 2 admits a Pareto improvement because there is a community (community-1 in the example) with more than one peaks of the members and its location is identical to the location for another community (community-2 in the example). Then by diversifying locations and regrouping communities, we can achieve a Pareto improvement. Community- $k$ in $N / \bar{k}$-decision $z \equiv\left(C_{k}, x_{k}\right)_{k=1}^{\bar{k}}$ is homogeneous if all members have the same peak, that is, $P\left(R_{C_{k}}\right)$ is a singleton. It is heterogeneous if it is not homogeneous. An $N / \bar{k}$-decision $z \equiv\left(C_{k}, x_{k}\right)_{k=1}^{\bar{k}}$ satisfies location diversity, if whenever there exists a heterogeneous community, say, $k \in\{1, \cdots, \bar{k}\}$, in $z$, its location $x_{k}$ differs from the location for any other community. There are situations where picking the same location for different communities is inevitable: for example, when all agents have the same peak but they are to be divided into more than one communities. Location diversity, then, has no bite.

Adding location diversity to no-envy and local stability, however, is not sufficient for efficiency as the next example shows.

Example 3. Let $N \equiv\{1,2,3,4\}$ and $\bar{k} \equiv 3$. Let $R \in \mathscr{R}^{N}$ be a profile such that $p\left(R_{1}\right)=$ $1, p\left(R_{2}\right)=2$, and $p\left(R_{3}\right)=p\left(R_{4}\right)=3$. Let $z \equiv\left(C_{k}, x_{k}\right)_{k=1}^{3}$ be defined as follows: $C_{1}=\{1,2\}, C_{2}=\{3\}, C_{3}=\{4\} \cdot x_{1} \equiv 1, x_{2}=x_{3} \equiv 3$. Note that $z$ satisfies no-envy, local stability, and location diversity. However, $z$ violates efficiency because there is a Pareto improvement $z^{\prime} \equiv\left(C_{k}^{\prime}, x_{k}^{\prime}\right)_{k=1}^{3}$ defined as follows: $C_{1}^{\prime}=\{1\}, C_{2}^{\prime}=\{2\}$, $C_{3}^{\prime}=\{3,4\}, x_{1}^{\prime}=1, x_{2}^{\prime}=2$, and $x_{3}^{\prime}=3$.

The $N / \bar{k}$-decision $z$ in Example 3 admits a Pareto improvement because there is a heterogeneous community (community-1 in the example) and at the same time, there are two other homogeneous communities with the same peaks (communities 2 and 3 in the example). Then after diversifying communities by combining members of the two homogeneous communities in $z$ and partitioning the heterogeneous community into two, we can achieve a Pareto improvement. Thus we need the following condition. An $N / \bar{k}$-decision $z \equiv\left(C_{k}, x_{k}\right)_{k=1}^{\bar{k}}$ satisfies community diversity, if whenever there is a heterogeneous community, there are no two other homogeneous communities with the same peak of their members, that is, for each pair of distinct homogeneous communities $k, l \in\{1, \ldots, \bar{k}\}, P\left(R_{C_{k}}\right) \neq P\left(R_{C_{l}}\right)$. When a decision satisfies both location diversity and community diversity, we say that it satisfies diversity.

Adding community diversity to the three conditions above, we finally get a necessary and sufficient condition for efficiency which is shown in our first result. The 
following lemmas are used in proving this result.

Throughout the lemmas, we fix $N \in \mathscr{N}, \bar{k} \in \mathbb{N}$, and $R \in \mathscr{R}^{N}$ and assume $\bar{k} \geq 2$.

Lemma 1. If an $N / \bar{k}$-decision $z \equiv\left(C_{k}, x_{k}\right)_{k=1}^{\bar{k}}$ satisfies no-envy, local efficiency, and location diversity at $R$, then

$$
p_{*}\left(R_{C_{1}}\right) \leq \cdots \leq p_{*}\left(R_{C_{\bar{k}}}\right) \text { and } p^{*}\left(R_{C_{1}}\right) \leq \cdots \leq p^{*}\left(R_{C_{\bar{k}}}\right) .
$$

Proof. To show $p_{*}\left(R_{C_{1}}\right) \leq \cdots \leq p_{*}\left(R_{C_{\bar{k}}}\right)$, let $k \in\{1, \cdots, \bar{k}-1\}$. Suppose, by contradiction, $p_{*}\left(R_{C_{k}}\right)>p_{*}\left(R_{C_{k+1}}\right)$. By local efficiency, $x_{k} \in P\left(R_{C_{k}}\right)$ and $x_{k+1} \in P\left(R_{C_{k+1}}\right)$. Since $x_{k} \leq x_{k+1}$, then $p_{*}\left(R_{C_{k+1}}\right)<x_{k+1}$ and $\left[p_{*}\left(R_{C_{k+1}}\right), x_{k+1}\right] \subseteq P\left(R_{C_{k+1}}\right)$. Hence $P\left(R_{C_{k+1}}\right)$ is not a singleton. Therefore, by location diversity, $x_{k}<x_{k+1}$. Then $p_{*}\left(R_{C_{k+1}}\right) \leq$ $x_{k}<x_{k+1}$. Hence, each agent $i \in C_{k+1}$ with $p\left(R_{i}\right) \leq x_{k}$ prefers $x_{k}$ to $x_{k+1}$, contradicting no-envy. The same argument can be used to prove the second inequalities.

Lemma 2. If an $N / \bar{k}$-decision $z \equiv\left(C_{k}, x_{k}\right)_{k=1}^{\bar{k}}$ satisfies no-envy, local efficiency, and location diversity at $R$, then $x_{1} \leq p_{*}\left(R_{C_{2}}\right), p^{*}\left(R_{\bar{k}-1}\right) \leq x_{\bar{k}}$, and for all $k$ with $2 \leq k \leq$ $\bar{k}-1$, (i) $p^{*}\left(R_{C_{k-1}}\right) \leq x_{k} \leq p_{*}\left(R_{C_{k+1}}\right)$; (ii) if $P\left(R_{C_{k}}\right)$ is not a singleton, $p^{*}\left(R_{C_{k-1}}\right)<$ $x_{k}<p_{*}\left(R_{C_{k+1}}\right)$; (iii) if $P\left(R_{C_{k-1}}\right)$ is not a singleton, $p^{*}\left(R_{C_{k-1}}\right)<x_{k}$; (iv) if $P\left(R_{C_{k+1}}\right)$ is not a singleton, $x_{k}<p_{*}\left(R_{C_{k+1}}\right)$; (v) if $P\left(R_{C_{k}}\right)$ is a singleton and $P\left(R_{C_{k-1}}\right)$ is not a singleton, then $x_{k}=p_{*}\left(R_{C_{k}}\right)=p^{*}\left(R_{C_{k}}\right)$ and $x_{k-1} \leq p^{*}\left(R_{C_{k-1}}\right)<x_{k}$.

Proof. We first show that for each $k \geq 2, p^{*}\left(R_{C_{k-1}}\right) \leq x_{k}$. Suppose, by contradiction, $x_{k}<p^{*}\left(R_{C_{k-1}}\right)$. Then there exists $i \in C_{k-1}$ such that $x_{k}<p\left(R_{i}\right)$. Since by local efficiency and Lemma $1, x_{k} \in P\left(R_{C_{k}}\right)$ and $x_{k}<p^{*}\left(R_{C_{k-1}}\right) \leq p^{*}\left(R_{C_{k}}\right)$, then $P\left(R_{C_{k}}\right)$ is not a singleton. Then by location diversity, $x_{k-1}<x_{k}$. Hence agent $i$ prefers $x_{k}$ to $x_{k-1}$, contradicting no-envy. Similarly, we show that for each $k \leq \bar{k}-1, x_{k} \leq p_{*}\left(R_{C_{k+1}}\right)$. This proves the first two inequalities and part (i). Parts (ii)-(v) are obtained easily from part (i), no-envy, and location diversity.

Lemma 3. Let $z \equiv\left(C_{k}, x_{k}\right)_{k=1}^{\bar{k}}$ be an $N / \bar{k}$-decision satisfying no-envy, local efficiency, and diversity at $R$. If there exists at least one heterogeneous community in $z$, then all locations are strictly ordered, that is, $x_{1}<x_{2}<\cdots<x_{\bar{k}}$.

Proof. Let $k \in\{1, \ldots, \bar{k}-1\}$. If at least one of $P\left(R_{C_{k}}\right)$ and $P\left(R_{C_{k+1}}\right)$ is not a singleton, then by Lemma 2 and local efficiency, $x_{k}<x_{k+1}$. Suppose both $P\left(R_{C_{k}}\right)$ and $P\left(R_{C_{k+1}}\right)$ are singleton. By local efficiency, $P\left(R_{C_{k}}\right)=\left\{x_{k}\right\}$ and $P\left(R_{C_{k+1}}\right)=\left\{x_{k+1}\right\}$. Then by community diversity, $P\left(R_{C_{k}}\right) \neq P\left(R_{C_{k+1}}\right)$. So $x_{k} \neq x_{k+1}$. Therefore $x_{k}<x_{k+1}$.

Lemma 4. Let $z \equiv\left(C_{k}, x_{k}\right)_{k=1}^{\bar{k}}$ be an $N / \bar{k}$-decision satisfying no-envy, local stability, and diversity at $R$. Assume that all locations in $z$ are strictly ordered. 
(i) If $z^{\prime} \equiv\left(C_{k}^{\prime}, x_{k}^{\prime}\right)_{k=1}^{\bar{k}}$ is another $N / \bar{k}$-decision such that for some $l_{0} \in\{1, \ldots, \bar{k}-1\}$, $x_{l_{0}}^{\prime}<x_{l_{0}}$ and for each $i \in C_{l_{0}}$, $z^{\prime} R_{i} z$, then there exist $l_{1}>l_{0}$ and $j_{1} \in C_{l_{0}}$ such that $j_{1} \in C_{l_{1}}^{\prime}$ and $x_{l_{1}}^{\prime}<x_{l_{1}}$.

(ii) If $z^{\prime} \equiv\left(C_{k}^{\prime}, x_{k}^{\prime}\right)_{k=1}^{\bar{k}}$ is another $N / \bar{k}$-decision such that for some $l_{0} \in\{2, \ldots, \bar{k}\}, x_{l_{0}}<$ $x_{l_{0}}^{\prime}$ and for each $i \in C_{l_{0}}, z^{\prime} R_{i} z$, then there exist $l_{1}<l_{0}$ and $j_{1} \in C_{l_{0}}$ such that $j_{1} \in C_{l_{1}}^{\prime}$ and $x_{l_{1}}<x_{l_{1}}^{\prime}$.

Proof. By local stability, there exists $j_{1} \in C_{l_{0}}$ such that $x_{l_{0}} \leq p\left(R_{j_{1}}\right)$ and for each $m \in\{1, \cdots, \bar{k}\}$, if $x_{m} \neq x_{l}$, then $x_{l_{0}} P_{j_{1}} x_{m}$. Since all locations in $z$ are strictly ordered, then for each $m \neq l_{0}, x_{l_{0}} P_{j_{1}} x_{m}$. For each $m \leq l_{0}$, since $x_{m}^{\prime} \leq x_{l_{0}}^{\prime}<x_{l_{0}} \leq p\left(R_{j_{1}}\right)$, then $x_{l_{0}}$ $P_{j_{1}} x_{m}^{\prime}$. Since $j_{1} \in C_{l_{0}}$ and $z^{\prime} R_{j_{1}} z$, then for each $m \leq l_{0}, j_{1} \notin C_{m}^{\prime}$. Therefore since $l_{0}<\bar{k}$, there exists $l_{1}>l_{0}$ such that $j_{1} \in C_{l_{1}}^{\prime}$. Since $l_{1}>l_{0}$ and $j_{1} \in C_{l_{0}}$, then by Lemma 2, $p\left(R_{j_{1}}\right) \leq x_{l_{1}}$. Since $x_{l_{1}}^{\prime} R_{j_{1}} x_{l_{0}}$ and $x_{l_{0}} P_{j_{1}} x_{l_{1}}$, then $x_{l_{1}}^{\prime}<x_{l_{1}}$. This completes the proof of part (i). The proof of part (ii) is similar.

Now we are ready to show the first result.

Theorem 1. A decision is efficient if and only if it satisfies no-envy, local stability, location diversity, and community diversity. And the four axioms are independent.

Proof. It is easy to prove that efficiency implies the four axioms, and so we omit this part. In what follows, we prove the converse. Throughout the proof, we fix $N \in \mathscr{N}$, $\bar{k} \in \mathbb{N}$, and $R \in \mathscr{R}^{N}$. Let $z \equiv\left(C_{k}, x_{k}\right)_{k=1}^{\bar{k}}$ be an $N / \bar{k}$-decision satisfying no-envy, local stability, and diversity. Suppose by contradiction that there exists a Pareto improvement of $z, z^{\prime} \equiv\left(C_{k}^{\prime}, x_{k}^{\prime}\right)_{k=1}^{\bar{k}}$. Let $i \in N$ be an agent who prefers $z^{\prime}$ to $z$. Let $k$ and $l_{0}$ be such that $i \in C_{k}$ and $i \in C_{l_{0}}^{\prime}$. Then $x_{k} \neq p\left(R_{i}\right)$ and $P\left(R_{C_{k}}\right)$ is not a singleton. Then by Lemma 3 , locations in $z$ are strictly ordered, that is,

$$
x_{1}<\cdots<x_{\bar{k}}
$$

Assume $p\left(R_{i}\right)<x_{k}$ (the symmetric argument applies for $x_{k}<p\left(R_{i}\right)$ ). We derive a contradiction for each of the following two cases.

Case 1. $l_{0} \geq k$. Since $x_{l_{0}}^{\prime} P_{i} x_{k}$ and $p\left(R_{i}\right)<x_{k}$, then $x_{l_{0}}^{\prime}<x_{k}$. Since $l_{0} \geq k, x_{k} \leq x_{l_{0}}$. Hence $x_{l_{0}}^{\prime}<x_{l_{0}}$. Since $\bar{k}$ is finite, then the iterative application of part (i) of Lemma 4 leads to $x_{\bar{k}}^{\prime}<x_{\bar{k}}$. By local stability, there exists $h \in C_{\bar{k}}$ such that $p\left(R_{h}\right) \geq x_{\bar{k}}$ and for each $m \leq \bar{k}$, if $x_{m} \neq x_{\bar{k}}$, then $x_{\bar{k}} P_{h} x_{m}$. Hence by (1), for each $m \neq \bar{k}, x_{\bar{k}} P_{h} x_{m}$. For each $m \leq \bar{k}$, since $x_{m}^{\prime} \leq x_{\bar{k}}^{\prime}<x_{\bar{k}} \leq p\left(R_{h}\right)$, then $x_{\bar{k}} P_{h} x_{m}^{\prime}$, contradicting the initial assumption.

Case 2. $l_{0}<k$. Since $i \in C_{k} \cap C_{l_{0}}^{\prime}$ and $z_{i}^{\prime} P_{i} z_{i}$, then $x_{l_{0}}^{\prime} P_{i} x_{k}$. By no-envy, $x_{k} R_{i}$ $x_{l_{0}}$. Since by (1), $x_{l_{0}}<x_{k}$ and $p\left(R_{i}\right)<x_{k}$, then $x_{l_{0}}<p\left(R_{i}\right)$. Therefore since $x_{l_{0}}^{\prime} P_{i} x_{l_{0}}$, 
$x_{l_{0}}<x_{l_{0}}^{\prime}$. Since $\bar{k}$ is finite, then the iterative application of part (ii) of Lemma 4 leads to $x_{1}<x_{1}^{\prime}$. By local stability, there exists $h \in C_{1}$ such that $p\left(R_{h}\right) \leq x_{1}$ and for each $m \leq \bar{k}$, if $x_{m} \neq x_{1}$, then $x_{1} P_{h} x_{m}$. Hence by (1), for each $m \neq 1, x_{1} P_{h} x_{m}$. For each $m \leq \bar{k}$, since $x_{m}^{\prime} \geq x_{1}^{\prime}>x_{1} \geq p\left(R_{h}\right)$, then $x_{1} P_{h} x_{m}^{\prime}$, contradicting the initial assumption.

Finally, independence of the four axioms is shown by the above three Examples 1-3 and Example 4 below.

Example 4. Let $N \equiv\{1, \cdots, 5\}$ and $\bar{k} \equiv 3$. Let $R$ be a profile such that $p\left(R_{1}\right)=0$, $p\left(R_{2}\right)=2, p\left(R_{3}\right)=p\left(R_{4}\right)=3$, and $p\left(R_{5}\right)=4$. Let $z \equiv\left(C_{k}, x_{k}\right)_{k=1}^{3}$ be the $N / \bar{k}$-decision defined as follows: $C_{1} \equiv\{1\}, C_{2} \equiv\{2,3\}, C_{3} \equiv\{3,4\}, x_{1} \equiv 0, x_{2} \equiv 3$, and $x_{3} \equiv 4$. Then $z$ satisfies local stability and diversity. However $z$ violates no-envy.

Theorem 1 enables us to check efficiency by testing the four elementary conditions, no-envy, local stability, location diversity, and community diversity. Checking efficiency can be simplified even further by using the following test.

Neighbor Test. For each $N \in \mathscr{N}$, each $\bar{k} \in \mathbb{N}$, and each $R \in \mathscr{R}^{N}, N / \bar{k}$-decision $z \equiv\left(C_{k}, x_{k}\right)_{k=1}^{\bar{k}}$ passes the neighbor test if it satisfies the following conditions. If $\left|\left\{p\left(R_{i}\right): i \in N\right\}\right| \leq \bar{k}$, then for each $k=1, \cdots, \bar{k}$ and each $i \in C_{k}, x_{k}=p\left(R_{i}\right)$. If $\left|\left\{p\left(R_{i}\right): i \in N\right\}\right|>\bar{k}$, then

(i) No overlap: For each $k \geq 2, p^{*}\left(R_{C_{k-1}}\right)<x_{k}$, and for each $k \leq \bar{k}-1, x_{k}<p_{*}\left(R_{C_{k+1}}\right)$;

(ii) Neighbor no-envy: For each $k$ with $2 \leq k \leq \bar{k}-1, x_{k} \in \bigcap_{i \in C_{k}} \operatorname{Max}\left[R_{i},\left\{x_{k-1}, x_{k}, x_{k+1}\right\}\right]$, $x_{1} \in \bigcap_{i \in C_{1}} \operatorname{Max}\left[R_{i},\left\{x_{1}, x_{2}\right\}\right]$, and $x_{\bar{k}} \in \bigcap_{i \in C_{\bar{k}}} \operatorname{Max}\left[R_{i},\left\{x_{\bar{k}-1}, x_{\bar{k}}\right\}\right]$, where for each $X \subseteq \mathbb{R}$, $\operatorname{Max}\left[R_{i}, X\right]$ is the set of all $R_{i}$-maximal elements in $X$;

(iii) Neighbor stability: For each $k \in\{1, \cdots, \bar{k}\}$, there exist $i, j \in C_{k}$ such that $p\left(R_{i}\right) \leq$ $x_{k} \leq p\left(R_{j}\right), x_{k} P_{i} x_{k-1}$, and $x_{k} P_{j} x_{k+1}$.

Theorem 2. A decision is efficient if and only if it passes the neighbor test.

Proof. Throughout the proof, we fix $N \in \mathscr{N}, \bar{k} \in \mathbb{N}$, and $R \in \mathscr{R}^{N}$ and consider the nontrivial case $\left|\left\{p\left(R_{i}\right): i \in N\right\}\right|>\bar{k}$. Let $z \equiv\left(C_{k}, x_{k}\right)_{k=1}^{\bar{k}}$ be an efficient $N / \bar{k}$-decision at $R$. Then by Theorem 1, $z$ satisfies no-envy, local stability, and diversity. By Lemma 2-(i), $x_{k} \leq p_{*}\left(R_{C_{k+1}}\right)$. Suppose $x_{k}=p_{*}\left(R_{C_{k+1}}\right)$. Note that by Lemma $3, x_{k}<x_{k+1}$. Thus by no-envy, $x_{k}<p_{*}\left(R_{C_{k+1}}\right)$. Similarly, we prove $p^{*}\left(R_{C_{k-1}}\right)<x_{k}$. Neighbor no-envy and neighbor stability follow directly from no-envy and local stability.

To prove the converse, let $z \equiv\left(C_{k}, x_{k}\right)_{k=1}^{\bar{k}}$ be an $N / \bar{k}$-decision passing the neighbor test. Note that by no-overlap,

$$
x_{1}<x_{2}<\cdots<x_{\bar{k}} \text {. }
$$


This implies location diversity and community diversity. To show no-envy, let $k$ be such that $2 \leq k \leq \bar{k}-1$. Let $i \in C_{k}$. By no-overlap, $x_{k-1}<p_{*}\left(R_{k}\right) \leq p\left(R_{i}\right)$. Therefore for all $l \leq k-1$, since $x_{l} \leq x_{k-1}$ and $x_{k} R_{i} x_{k-1}$ by neighbor no-envy, then $x_{k} R_{i} x_{l}$. Also by no-overlap, $p\left(R_{i}\right) \leq p^{*}\left(R_{k}\right)<x_{k+1}$. For all $l \geq k+1$, since $x_{l} \geq x_{k+1}$ and $x_{k} R_{i} x_{k+1}$ by neighbor no-envy, then $x_{k} R_{i} x_{l}$. Applying the same argument for $k=1$ and $k=\bar{k}$, we show that $z$ satisfies no-envy. For each $k=1, \cdots, \bar{k}$, by neighbor stability, there exist $i, j \in C_{k}$ such that $p\left(R_{i}\right) \leq x_{k} \leq p\left(R_{j}\right), x_{k} P_{i} x_{k-1}$, and $x_{k} P_{j} x_{k+1}$. Then by (2), for each $l \neq k, x_{k} P_{i} x_{l}$ and $x_{k} P_{j} x_{l}$. Therefore $i, j \in C_{k}^{o}(R, z)$. Since $p\left(R_{i}\right) \leq x_{k} \leq p\left(R_{j}\right)$, $x_{k} \in P\left(R_{C_{k}^{o}(R, z)}\right)$. Hence $z$ satisfies local stability. Therefore, by Theorem 1 , $z$ satisfies efficiency.

When $\bar{k}=2$, the neighbor test reduces to a substantially simpler condition as stated in Miyagawa (2001).

\subsection{The Game of Community Division and Location}

In this section, we consider a simple game similar to the local public good provision game considered by Konishi, Le Breton, and Weber (1998).

Definition 1 (The Game of Community Division and Location). Given a set of agents $N \in \mathscr{N}$ and a required number of communities $\bar{k} \in \mathbb{N}$, let $G$ be a game form with the following strategy sets and the outcome function. For each $i \in N$, let $S_{i} \equiv \mathbb{R}$ be $i$ 's strategy set with generic element $s_{i}$, interpreted as $i$ 's proposal for the location of the local public facility in his community. Let $S \equiv \times_{i \in N} S_{i}$ be the set of strategy profiles with generic element $s$. When the total number of locations proposed by agents at $s$ is less than or equal to the required level $\bar{k}$, agents with the same proposals constitute communities and locations are determined by their proposals. Otherwise, communities are formed in the same way but no public facility can be provided for any community. Formally, let $g: S \rightarrow Z$ be the outcome function defined as follows: for each strategy profile $s \equiv\left(s_{i}\right)_{i \in N}$,

(i) if $k(s) \equiv\left|\left\{s_{i}: i \in N\right\}\right| \leq \bar{k}$, let $g(s)$ be the decision $\left(C_{k}, x_{k}\right)_{k=1}^{k(s)}$ such that $\left\{C_{k}\right\}_{k=1}^{k(s)}$ is a partition of $N$ and for each $k=1, \cdots, k(s), C_{k} \equiv\left\{i \in N: s_{i}=x_{k}\right\}$,

(ii) if $k(s) \equiv\left|\left\{s_{i}: i \in N\right\}\right|>\bar{k}$, let $g(s)$ be the decision $\left(C_{k}, v\right)_{k=1}^{k(s)}$ such that $\left\{C_{k}\right\}_{k=1}^{k(s)}$ is a partition of $N$ and for each $k=1, \cdots, k(s), C_{k} \equiv\left\{i \in N: s_{i}=x_{k}\right\}$.

A strategy profile $s \in S$ is a strong Nash equilibrium in $(G, R)$, if there exist no $T \subseteq N$ and $s_{T}^{\prime} \in S_{T}$ such that for each $i \in T, g\left(s_{T}^{\prime}, s_{-T}\right) R_{i} g(s)$ and for some $j \in$ $T, g\left(s_{T}^{\prime}, s_{-T}\right) P_{j} g(s){ }^{3}$ The next result is that the set of efficient decisions can be

\footnotetext{
${ }^{3}$ The definition is from Moulin (1994). Aumann (1959) introduces a weaker version of strong Nash
} 
implemented in strong Nash equilibrium by this game form $G$. Theorem 1 plays a crucial role in proving this result.

Theorem 3. Let $N \in \mathscr{N}$ and $\bar{k} \in \mathbb{N}$. For each $R \in \mathscr{R}^{N}$, an $N / \bar{k}$-decision is efficient if and only if it is a strong Nash equilibrium outcome of the game of community division and location.

Proof. Let $R \in \mathscr{R}^{N}$ and $\bar{k} \in \mathbb{N}$ be given. By definition, every strong Nash equilibrium outcome is efficient. In order to prove the converse, let $z \equiv\left(C_{k}, x_{k}\right)_{k=1}^{\vec{k}}$ be an efficient $N / \bar{k}$-decision. Then by Theorem $1, z$ satisfies no-envy, local stability, and diversity. Let $s$ be such that for each $k \in\{1, \cdots, \bar{k}\}$ and each $i \in C_{k}, s_{i} \equiv x_{k}$. We only have to show that $s$ is a strong Nash equilibrium. When $\left|\left\{p\left(R_{i}\right): i \in N\right\}\right| \leq \bar{k}$, every agent gets his peak location and so $s$ is, clearly, a strong Nash equilibrium. Assume that $\mid\left\{p\left(R_{i}\right)\right.$ : $i \in N\} \mid>\bar{k}$. Then there exists a heterogeneous community. Hence by Lemma 3, $x_{1}<x_{2}<\cdots<x_{\bar{k}}$. Suppose by contradiction that there exist $T \subseteq N$ and $s_{T}^{\prime}$ such that for all $i \in T, g\left(s_{T}^{\prime}, s_{-T}\right) R_{i} z$ and for some $j \in T, g\left(s_{T}^{\prime}, s_{-T}\right) P_{j} z$. Let $z^{\prime} \equiv g\left(s_{T}^{\prime}, s_{-T}\right)$. Since $v$ is the worst outcome for everyone,

$$
\left|\left\{s_{i}^{\prime}: i \in T\right\} \cup\left\{s_{j}: j \in N \backslash T\right\}\right| \leq \bar{k}
$$

For each $k \in\{1, \cdots, \bar{k}\}$, let $T_{k} \equiv T \cap C_{k}$.

Claim 1. There exists $k \in\{1, \cdots, \bar{k}\}$ such that $T_{k}=C_{k}$.

Proof. Suppose by contradiction that for each $k \in\{1, \cdots, \bar{k}\}, T_{k} \neq C_{k}$. Then $\mid\left\{s_{i}^{\prime}\right.$ : $i \in T\} \cup\left\{s_{j}: j \in N \backslash T\right\} \mid \geq \bar{k}$. Therefore, by (3), $\left|\left\{s_{i}^{\prime}: i \in T\right\} \cup\left\{s_{j}: j \in N \backslash T\right\}\right|=\bar{k}$. Then $\left\{s_{i}^{\prime}: i \in T\right\} \cup\left\{s_{j}: j \in N \backslash T\right\}=\left\{x_{1}, \cdots, x_{\bar{k}}\right\}$. Therefore, since $z$ satisfies no-envy, there is no $i \in T$ such that $z^{\prime} P_{i} z$, contradicting the previous assumption on $T$ and $s_{T}^{\prime}$.

Since $\left\{k \in\{1, \cdots, \bar{k}\}: T_{k}=C_{k}\right\} \neq \emptyset$, we may write this set as $\left\{k_{1}, \cdots, k_{\bar{r}}\right\}$ for some $\bar{r} \geq 1$ where $k_{1}, \cdots, k_{\bar{r}} \in\{1, \cdots, \bar{k}\}$ are such that $k_{1}<\cdots<k_{\bar{r}}$.

Claim 2. (i) There exist $i_{1}, \cdots, i_{\bar{r}} \in N$ such that for each $r \in\{1, \cdots, \bar{r}\}, i_{r} \in T_{k_{r}} \equiv$ $C_{k_{r}}, s_{i_{r}}^{\prime} \leq x_{k_{r}}, s_{i_{r}}^{\prime} \notin\left\{x_{k}: k \notin\left\{k_{1}, \cdots, k_{\bar{r}}\right\}\right\}$, and $s_{i_{1}}^{\prime}<s_{i_{2}}^{\prime}<\cdots<s_{i_{\bar{r}}}^{\prime}$.

(ii) There exist $j_{1}, \cdots, j_{\bar{r}} \in N$ such that for each $r \in\{1, \cdots, \bar{r}\}, j_{r} \in T_{k_{r}} \equiv C_{k_{r}}, s_{j_{r}}^{\prime} \geq x_{k_{r}}$, $s_{j_{r}}^{\prime} \notin\left\{x_{k}: k \notin\left\{k_{1}, \cdots, k_{\bar{r}}\right\}\right\}$, and $s_{j_{1}}^{\prime}<s_{j_{2}}^{\prime}<\cdots<s_{j_{\bar{r}}}^{\prime}$.

Proof. By (3), each $i \in N$ eventually gets a location that is chosen by himself. Therefore for each $r$ and each $i \in T_{k_{r}}, s_{i}^{\prime} R_{i} x_{k_{r}}$. Let $r \in\{1, \cdots, \bar{r}\}$. By local stability, there exist $i_{r}, j_{r} \in C_{k_{r}}^{0}(R, z)$ such that $p\left(R_{i_{r}}\right) \leq x_{k_{r}} \leq p\left(R_{j_{r}}\right)$. Clearly, $s_{i_{r}}^{\prime} \leq x_{k_{r}} \leq s_{j_{r}}^{\prime}$.

equilibrium. Konishi, Le Breton, and Weber (1999) study the relationship between strong Nash equilibrium and "coalition-proof Nash equilibrium" in the context of common agency games. 
Note that for all $k \neq k_{r}, x_{k_{r}} P_{j_{r}} x_{k}$ and $x_{k_{r}} P_{i_{r}} x_{k}$. Also note that by Lemma 2, if $k>k_{r}$, $p\left(R_{j_{r}}\right) \leq x_{k}$. Therefore for all $r, s_{j_{r}}^{\prime}<s_{j_{r+1}}^{\prime}$ and $s_{j_{r}} \notin\left\{x_{k}: k \notin\left\{k_{1}, \cdots, k_{\bar{r}}\right\}\right\}$. Similarly, for all $r, s_{i_{r}}^{\prime}<s_{i_{r+1}}^{\prime}$ and $s_{i_{r}} \notin\left\{x_{k}: k \notin\left\{k_{1}, \cdots, k_{\bar{r}}\right\}\right\}$.

Claim 3. There exists $r \in\{1, \cdots, \bar{r}\}$ such that $s_{i_{r}}^{\prime} \neq x_{k_{r}}$.

Proof. Suppose by contradiction that for all $r \in\{1, \cdots, \bar{r}\}, s_{i_{r}}^{\prime}=x_{k_{r}}$. Then at $z^{\prime}$, each agent in $T$ gets a location that is a location in $z$. Therefore, since $z$ satisfies noenvy, then there is no $i \in T$ such that $z^{\prime} P_{i} z$, contradicting the earlier assumption on $T$ and $s_{T}^{\prime}$.

To complete the proof, let $r$ be such that $s_{i_{r}}^{\prime} \neq x_{k_{r}}$. Then by Claim 2, $s_{i_{r}}^{\prime}<s_{j_{r}}^{\prime}$. Therefore, by Claim 3, $\left\{x_{k}: k \notin\left\{k_{1}, \cdots, k_{\bar{r}}\right\}\right\} \cup\left\{s_{i_{r}}^{\prime}, s_{j_{r}}^{\prime}\right\} \cup\left\{s_{i_{1}}^{\prime}, \cdots, s_{i_{r-1}}^{\prime}\right\} \cup\left\{s_{j_{r+1}}^{\prime}, \cdots, s_{j_{\bar{r}}}^{\prime}\right\}$ is composed of more than $\bar{k}$ elements, contradicting (3).

\section{Self-Selection Consistent Social Choice}

In this section, we provide an algorithm that can be used to construct a large family of efficient and consistent, namely self-selection consistent, social choice functions.

We first give a simple characterization of consistent social choice functions. A single location function $f: \cup_{N \in \mathscr{N}} \mathscr{R}^{N} \rightarrow \mathbb{R}$ maps each single location problem (a preferences profile) into a location. Examples are the minimal peak function $f^{\min }(\cdot)$ and the mean peak function $f^{\text {mean }}(\cdot)$ defined as follows: for each $N \in \mathscr{N}$ and each $R \in \mathscr{R}^{N}, f^{\min }(R) \equiv \min \left\{p\left(R_{i}\right): i \in N\right\}$ and $f^{\text {mean }}(R) \equiv \sum_{i \in N} p\left(R_{i}\right) /|N|$.

Proposition 1. A social choice function $\varphi(\cdot)$ satisfies consistency if and only if there is a unique single location function $f(\cdot)$ such that for each $(R, \bar{k}) \in \mathscr{D}$ and each $k=$ $1, \ldots, \bar{k}, x_{k}=f\left(R_{C_{k}}\right)$, where $\left(C_{k}, x_{k}\right)_{k=1}^{\bar{k}}=\varphi(R, \bar{k})$.

Proof. The proof for the "if" part is evident. In order to prove the "only if" part, let $\varphi(\cdot)$ be a consistent social choice function. Let $f(\cdot) \equiv \varphi^{L}(\cdot, 1)$. Let $(R, \bar{k}) \in \mathscr{D}$. Let $\left(C_{k}, x_{k}\right)$ be a pair of a community and the location for its public facility chosen by $\varphi(\cdot)$ for $(R, \bar{k})$. Then by consistency, $x_{k}=\varphi^{L}\left(R_{C_{k}}, 1\right)$. So $x_{k}=f\left(R_{C_{k}}\right)$. Uniqueness of $f(\cdot)$ follows from the fact that any single location function $f(\cdot)$ with the stated condition should satisfy $f(\cdot)=\varphi^{L}(\cdot, 1)$.

The next example shows existence of an efficient and consistent social choice function using the minimal peak function $f^{\min }(\cdot)$.

Example 5 (An efficient and consistent function). For each $N \in \mathscr{N}$ and each $(R, \bar{k}) \in$ $\mathscr{D}^{N}$ with $\left|\left\{p\left(R_{i}\right): i \in N\right\}\right| \geq \bar{k}$, let $p_{1}<p_{2}<\cdots<p_{\bar{k}}$ be the list of the $\bar{k}$ smallest peaks. For each $k \in\{1, \ldots, \bar{k}\}$, if $k<\bar{k}, C_{k} \equiv\left\{i \in N: p\left(R_{i}\right)=p_{k}\right\}$ and if $k=\bar{k}, C_{\bar{k}} \equiv$ 
$\left\{i \in N: p\left(R_{i}\right) \geq p_{\bar{k}}\right\}$. Let $\varphi_{k}(R, \bar{k}) \equiv\left(C_{k}, f^{\min }\left(R_{C_{k}}\right)\right)$. It is easily shown by Theorem 2 and Proposition 1 that $\varphi(\cdot)$ satisfies both efficiency and consistency.

It can be shown that alternative constructions obtained by replacing $f^{\min }(\cdot)$ with some other single location functions, e.g. $f^{\text {mean }}(\cdot)$, may not give an efficient social choice function.

In what follows, we provide an algorithm that will allow us to construct an efficient and consistent function based on any single location function with the following two mild conditions. A single location function $f$ satisfies efficiency if for each $N \in \mathscr{N}$ and each $R \in \mathscr{R}^{N}, f(R) \in P(R)$. It satisfies participation if for each $N \in \mathscr{N}$, each $i \notin N$, and each $R \in \mathscr{R}^{N \cup\{i\}}, f(R) R_{i} f\left(R_{-i}\right)$ (Barberà and Beviá 2002).

Leftward Adjustment Algorithm. ${ }^{4}$ Let $N \in \mathscr{N}, R \in \mathscr{R}^{N}$, and $\bar{k} \in \mathbb{N}$. Let $z$ be an $N / \bar{k}$ decision and $f(\cdot)$ a single location function. To simplify our notation, for each $S \subseteq N$, we use $f(S)$ instead of $f\left(R_{S}\right)$.

Stage 1. Let $l \equiv \max \left\{k: k=1\right.$ or when $\left.k \geq 2, C_{k}^{*} \neq \emptyset\right\}$, where $C_{k}^{*} \equiv\left\{i \in C_{k}\right.$ : $\left.x_{k-1} R_{i} x_{k}\right\} \neq \emptyset$.

Stage 2. If $l=1$, go to Stage 8. If not, for each $k<l-1$, let $z_{k}^{\prime} \equiv z_{k}$ and go to Stage 3 .

Stage 3. Let $\hat{C}_{l-1} \equiv C_{l-1} \cup C_{l}^{*}$ and go to Stage 4 .

Stage 4. Let $k \equiv l-1$ and go to Stage 5.1.

Stage 5.1. If there is $i \in C_{k+1} \backslash \hat{C}_{k}$ such that $p_{i} \leq f\left(\hat{C}_{k}\right)$, go to Stage 5.1.1. If not, go to Stage 5.2.

Stage 5.1.1. Pick $i \in C_{k+1} \backslash \hat{C}_{k}$ such that

$$
\min \left\{y \in X: y I_{i} f\left(\hat{C}_{k}\right)\right\}=\min \left\{\min \left\{y: y I_{h} f\left(\hat{C}_{k}\right)\right\}: h \in C_{k+1} \backslash \hat{C}_{k} \text { and } p_{h} \leq f\left(\hat{C}_{k}\right)\right\}
$$

and go to Stage 5.1.2.

Stage 5.1.2. Put $\hat{C}_{k}==\hat{C}_{k} \cup\{i\}$ and return to Stage 5.1.

Stage 5.2. Let $C_{k}^{\prime} \equiv \hat{C}_{k}$ and $x_{k}^{\prime} \equiv f\left(C_{k}^{\prime}\right)$. And go to Stage 5.3.

Stage 5.3. If $k+1<\bar{k}$, go to Stage 5.4. If not, go to Stage 6 .

Stage 5.4. Put $k==k+1$ and go to Stage 5.5.

Stage 5.5. Let $\hat{C}_{k} \equiv C_{k} \backslash C_{k-1}^{\prime}$ and return to Stage 5.1.

Stage 6. Let $C_{\bar{k}}^{\prime} \equiv C_{\bar{k}} \backslash C_{\bar{k}-1}^{\prime}$ and $x_{\bar{k}}^{\prime} \equiv f\left(C_{\bar{k}}^{\prime}\right)$. Then go to Stage 7 .

Stage 7. Put $z==z^{\prime}$ and return to Stage 1 .

Stage 8. The algorithm ends with $z$.

\footnotetext{
${ }^{4}$ Our algorithm is a modification of the one provided in the proof of Proposition 1 by Barberà and Beviá (2002). Reversing the direction of moving agents within the leftward adjustment algorithm, we can define the "rightward adjustment algorithm" and establish the same result as we do later for the leftward adjustment algorithm.
} 


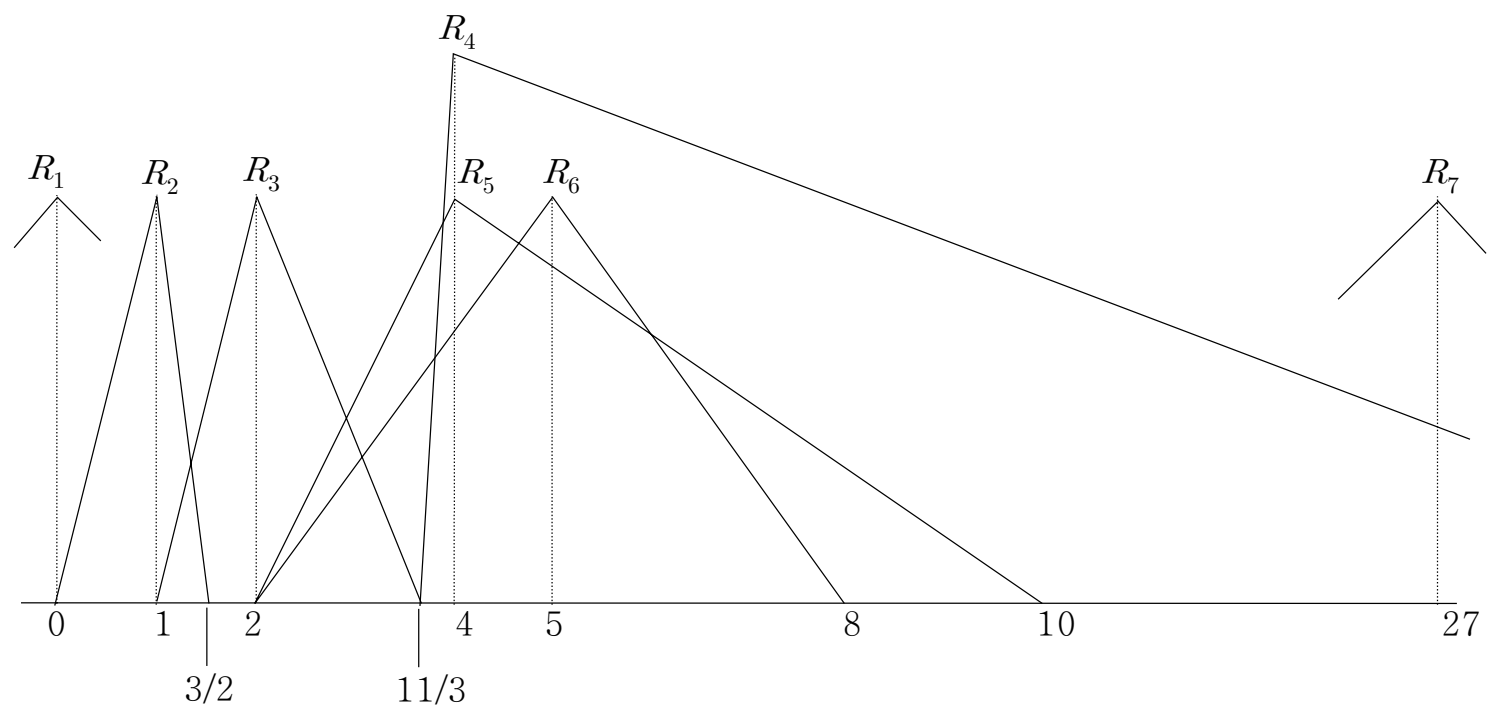

Figure 1: Let $N \equiv\{1, \ldots, 7\}$ and $\bar{k} \equiv 4$. Consider the preference profile $R$ of agents in $N$ depicted in the figure. The leftward adjustment algorithm with initial decision $((\{1\}, 0),(\{2\}, 1),(\{3\}, 2),(\{4,5,6,7\}, 10))$ leads to four iterations of loop 3, as explained in Tables 1-4, and yields the following efficient final decision: $((\{1,2\}, 1 / 2),(\{3\}, 2),(\{4,5,6\}, 13 / 3),(\{7\}, 27))$.

We refer to the combination of Stages 5.1, 5.1.1, and 5.1.2 as loop 1, the combination of loop 1 and Stages 5.2-5.5 as loop 2 and the combination of Stages 1-7 and loops 1 and 2 as loop 3.

Convergence of this algorithm depends on what initial $N / \bar{k}$-decision is used. Proposition 2 below states conditions for convergence and, in addition, convergence to an efficient decision.

Proposition 2. Assume that $f(\cdot)$ is a single location function satisfying efficiency and participation. Let $N \in \mathscr{N}, R \in \mathscr{R}^{N}$, and $\bar{k} \in \mathbb{N}$. Let $z \equiv\left(C_{k}, x_{k}\right)_{k=1}^{\bar{k}}$ be an $N / \bar{k}$-decision such that for each $k=1, \cdots, \bar{k}, x_{k}=f\left(R_{C_{k}}\right)$. If $z$ satisfies no-overlap and for each $k=1, \cdots, \bar{k}-1$,

$$
\begin{aligned}
& \text { No-right-envy: } x_{k} R_{i} x_{k+1} \text { for each } i \in C_{k} \text {; } \\
& \text { Right stability: } x_{k} P_{j} x_{k+1} \text { and } p\left(R_{j}\right) \geq x_{k} \text { for some } j \in C_{k} \text {, }
\end{aligned}
$$

then the leftward adjustment of $z$ based on $f$ converges to an efficient $N / \bar{k}$-decision. Proof. See the appendix.

To better understand how the algorithm works, consider the following example: 


\begin{tabular}{|c|c|c|c|c|}
\hline Loop 3 \#1 & Community 1 & Community 2 & Community 3 & Community 4 \\
\hline Initiondecicion & $\{1\}$ & $\{2\}$ & $\{3\}$ & $\{4,5,6,7\}$ \\
\hline initial decision & 0 & 1 & 2 & 10 \\
\hline Stage 1 & & $C_{2}^{*}=\emptyset$ & $C_{3}^{*}=\emptyset$ & $C_{4}^{*}=\{5,6\}$ \\
\hline Stage 2 & $\begin{array}{c}C_{1}^{\prime}=\{1\} \\
x_{1}^{\prime}=0\end{array}$ & $\begin{array}{c}C_{2}^{\prime}=\{2\} \\
x_{2}^{\prime}=1\end{array}$ & & \\
\hline Stage 3 & & & $\begin{array}{l}\hat{C}_{3}=\{3,5,6\} \\
f\left(\hat{C}_{3}\right)=11 / 3\end{array}$ & \\
\hline Stages 4 and 5.1 & & & & \\
\hline Stage 5.2 & & & $\begin{array}{c}C_{3}^{\prime}=\{3,5,6\} \\
x_{3}^{\prime}=11 / 3\end{array}$ & \\
\hline Stages 5.3-5.5 & & & & \\
\hline Stage 6 & & & & $\begin{aligned} C_{4}^{\prime} & =\{4,7\} \\
x_{4}^{\prime} & =31 / 2\end{aligned}$ \\
\hline Stage 7 & $\begin{array}{c}\{1\} \\
0\end{array}$ & $\begin{array}{c}\{2\} \\
1\end{array}$ & $\begin{array}{c}\{3,5,6\} \\
11 / 3\end{array}$ & $\begin{array}{l}\{4,7\} \\
31 / 2\end{array}$ \\
\hline
\end{tabular}

Table 1: The first iteration of loop 3 in Example 6. The set in each cell gives composition of the corresponding community and the real number in each cell is the location for the community. The first row gives the initial decision and the last row gives the final decision of this iteration.

Example 6. Let $N \equiv\{1, \ldots, 7\}$ and $\bar{k}=4$. Let $R_{1}, \ldots, R_{7}$ be such that $p\left(R_{1}\right)=0$, $p\left(R_{2}\right)=1, p\left(R_{3}\right)=2, p\left(R_{4}\right)=p\left(R_{5}\right)=4, p\left(R_{6}\right)=5, p\left(R_{7}\right)=27$ and $0 I_{2} 3 / 2$, $1 I_{3} 11 / 3,31 / 2 P_{4} 11 / 3,2 I_{5} 10,2 P_{6} 10$ as illustrated in Figure 1 . Let $f(\cdot) \equiv f^{\text {mean }}(\cdot)$. Consider the initial decision of

$$
((\{1\}, 0),(\{2\}, 1),(\{3\}, 2),(\{4,5,6,7\}, 10))
$$

satisfying no-right-envy and right stability. The leftward adjustment of this initial decision consists of four iterations of loop 3, as explained in Tables 1-4. The algorithm yields

$$
((\{1,2\}, 1 / 2),(\{3\}, 2),(\{4,5,6\}, 13 / 3),(\{7\}, 27)) .
$$

The first iteration of loop 3: It is explained in Table 1 and yields the following decision:

$$
((\{1\}, 0),(\{2\}, 1),(\{3,5,6\}, 11 / 3),(\{4,7\}, 31 / 2)) .
$$

In this process, neither loop 1 nor loop 2 is triggered as explained by blank cells in rows 6 and 8 of Table 1 .

The second iteration of loop 3: It is explained in Table 2 and starts with the decision 


\begin{tabular}{|c|c|c|c|c|}
\hline Loop 3 \#2 & Community 1 & Community 2 & Community 3 & Community 4 \\
\hline \multirow{2}{*}{ Initial decision } & $\{1\}$ & $\{2\}$ & $\{3,5,6\}$ & $\{4,7\}$ \\
\hline & 0 & 1 & $11 / 3$ & $31 / 2$ \\
\hline Stage 1 & & $C_{2}^{*}=\emptyset$ & $C_{3}^{*}=\{3\}$ & $C_{4}^{*}=\emptyset$ \\
\hline Stage 2 & $\begin{array}{c}C_{1}^{\prime}=\{1\} \\
x_{1}^{\prime}=0\end{array}$ & & & \\
\hline Stage 3 & & $\begin{array}{l}\hat{C}_{2}=\{2,3\} \\
f\left(\hat{C}_{2}\right)=3 / 2\end{array}$ & & \\
\hline \multicolumn{5}{|l|}{ Stages 4-5.1 } \\
\hline Stage 5.2 & & $\begin{array}{c}C_{2}^{\prime}=\{2,3\} \\
x_{2}^{\prime}=3 / 2\end{array}$ & & \\
\hline Stages 5.3-5.5 & & & $\begin{array}{c}\hat{C}_{3}=\{5,6\} \\
f\left(\hat{C}_{3}\right)=9 / 2\end{array}$ & \\
\hline Stages 5.1-5.1.2 & & & $\begin{array}{l}\hat{C}_{3}=\{4,5,6\} \\
f\left(\hat{C}_{3}\right)=13 / 3\end{array}$ & \\
\hline \multicolumn{5}{|l|}{ Stage 5.1} \\
\hline Stage 5.2 & & & $\begin{array}{c}C_{3}^{\prime}=\{4,5,6\} \\
x_{3}^{\prime}=13 / 3\end{array}$ & \\
\hline \multicolumn{5}{|l|}{ Stage 5.3} \\
\hline Stage 6 & & & & $\begin{aligned} C_{4}^{\prime} & =\{7\} \\
x_{4}^{\prime} & =27\end{aligned}$ \\
\hline Stage 7 & $\begin{array}{c}\{1\} \\
0\end{array}$ & $\begin{array}{c}\{2,3\} \\
3 / 2\end{array}$ & $\begin{array}{c}\{4,5,6\} \\
13 / 3\end{array}$ & $\begin{array}{l}\{7\} \\
27\end{array}$ \\
\hline
\end{tabular}

Table 2: The second iteration of loop 3 in Example 6.

obtained after the first iteration. It yields

$$
((\{1\}, 0),(\{2,3\}, 3 / 2),(\{4,5,6\}, 13 / 3),(\{7\}, 27)) .
$$

Loop 2 is triggered once because $l=3<\bar{k}(=4)$ as explained in rows 8-11. Loop 1 is triggered within the first iteration of loop 2 , which is explained in row 9.

The third iteration of loop 3: It is explained in Table 3 and yields

$$
((\{1,2\}, 1 / 2),(\{3\}, 2),(\{4,5,6\}, 13 / 3),(\{7\}, 27)) .
$$

In this process, loop 2 is triggered twice in rows 8-10 first and rows 11-13 second. However, loop 1 is not triggered as explained by blank cells in rows 6, 9, and 12 .

The fourth iteration of loop 3: It is explained in Table 4 and starts with the decision from the third iteration. Note that at this decision, no agent weakly prefers the location for the left adjacent community to the location for his own community, which means 


\begin{tabular}{|c|c|c|c|c|}
\hline Loop 3 \#3 & Community 1 & Community 2 & Community 3 & Community 4 \\
\hline \multirow{2}{*}{ Initial Decision } & $\{1\}$ & $\{2,3\}$ & $\{4,5,6\}$ & $\{7\}$ \\
\hline & 0 & $3 / 2$ & $13 / 3$ & 27 \\
\hline Stage 1 & & $C_{2}^{*}=\{2\}$ & $C_{3}^{*}=\emptyset$ & $C_{4}^{*}=\emptyset$ \\
\hline \multicolumn{5}{|l|}{ Stage 2} \\
\hline Stage 3 & $\begin{array}{l}\hat{C}_{1}=\{1,2\} \\
f\left(\hat{C}_{1}\right)=1 / 2\end{array}$ & & & \\
\hline \multicolumn{5}{|l|}{ Stages 4-5.1 } \\
\hline Stage 5.2 & $\begin{array}{c}C_{1}^{\prime}=\{1,2\} \\
x_{1}^{\prime}=1 / 2\end{array}$ & & & \\
\hline Stages 5.3-5.5 & & $\begin{array}{l}\hat{C}_{2}=\{3\} \\
f\left(\hat{C}_{2}\right)=2\end{array}$ & & \\
\hline \multicolumn{5}{|l|}{ Stage 5.1} \\
\hline Stage 5.2 & & $\begin{array}{c}C_{2}^{\prime}=\{3\} \\
x_{2}^{\prime}=2\end{array}$ & & \\
\hline Stage 5.3-5.5 & & & $\begin{array}{l}\hat{C}_{3}=\{4,5,6\} \\
f\left(\hat{C}_{3}\right)=13 / 3\end{array}$ & \\
\hline \multicolumn{5}{|l|}{ Stage 5.1} \\
\hline Stage 5.2 & & & $\begin{array}{c}C_{3}^{\prime}=\{4,5,6\} \\
x_{3}^{\prime}=13 / 3\end{array}$ & \\
\hline \multicolumn{5}{|l|}{ Stage 5.3} \\
\hline Stage 6 & & & & $\begin{aligned} C_{4}^{\prime} & =\{7\} \\
x_{4}^{\prime} & =27\end{aligned}$ \\
\hline Stage 7 & $\begin{array}{c}\{1,2\} \\
1 / 2\end{array}$ & $\begin{array}{c}\{3\} \\
2\end{array}$ & $\begin{array}{c}\{4,5,6\} \\
13 / 3\end{array}$ & $\begin{array}{l}\{7\} \\
27\end{array}$ \\
\hline
\end{tabular}

Table 3: The third iteration of loop 3 in Example 6.

$l=1$. Thus this decision is the final outcome of the algorithm.

For each $(R, \bar{k}) \in \mathscr{D}$, let $L A^{f}(R, \bar{k})$ be the set of all $N / \bar{k}$-decisions $z \equiv\left(C_{k}, x_{k}\right)_{k=1}^{\bar{k}}$ with no-overlap, no-right-envy, and right stability such that for each $k=1, \cdots, \bar{k}$, $x_{k}=f\left(R_{C_{k}}\right)$. It is easy to show that for each $(R, \bar{k}) \in \mathscr{D}, L A^{f}(R, \bar{k}) \neq \emptyset .^{5}$ Using any decision in $L A^{f}(R, \bar{k})$ as the initial decision in the leftward adjustment algorithm and associating with $(R, \bar{k})$ an outcome of the algorithm, we can define a self-selection

\footnotetext{
${ }^{5}$ Barberà and Beviá (2002) shows this by the following construction. If the number of peaks is less than $\bar{k}$, the proof is trivial. Suppose $\left|\left\{p\left(R_{i}\right): i \in N\right\}\right| \geq \bar{k}$. Let $r$ be the number of peaks $(r \geq \bar{k})$. Denote peaks in $\left\{p\left(R_{i}\right): i \in N\right\}$ in the increasing order by $p_{1}<\cdots<p_{r}$. Then we can partition $N$ into $\bar{k}$ communities $\left(C_{1}, \cdots, C_{\bar{k}}\right)$ as follows: $C_{1} \equiv\left\{i \in N: p\left(R_{i}\right)=p_{1}\right\}, C_{2} \equiv\left\{i \in N: p\left(R_{i}\right)=p_{2}\right\}$, $\cdots, C_{\bar{k}-1} \equiv\left\{i \in N: p\left(R_{i}\right)=p_{\bar{k}-1}\right\}, C_{\bar{k}} \equiv\left\{i \in N: p\left(R_{i}\right) \geq p_{\bar{k}}\right\}$. Let $x_{1} \equiv p_{1}, \cdots, x_{\bar{k}-1} \equiv p_{\bar{k}-1}$, and $x_{\bar{k}} \equiv f\left(R_{C_{\bar{k}}}\right)$. Let $z \equiv\left(C_{k}, x_{k}\right)_{k=1}^{\bar{k}}$. Every member in community- $k$, for each $k=1, \cdots, \bar{k}-1$, gets his peak location and so both (4) and (5) hold. Since $f$ satisfies efficiency and each community- $k$ with $k \leq \bar{k}-1$ has only one peak, $f\left(R_{C_{k}}\right)=p_{k}\left(=x_{k}\right)$. Finally, no-overlap holds by construction of $z$.
} 


\begin{tabular}{|l|c|c|c|c|}
\hline \multicolumn{1}{|c|}{ Loop 3 \#4 } & Community 1 & Community 2 & Community 3 & Community 4 \\
\hline \multirow{2}{*}{ Initial Decision } & $\{1,2\}$ & $\{3\}$ & $\{4,5,6\}$ & $\{7\}$ \\
& $1 / 2$ & 2 & $13 / 3$ & 27 \\
\hline Stage 1 & & $C_{2}^{*}=\emptyset$ & $C_{3}^{*}=\emptyset$ & $C_{4}^{*}=\emptyset$ \\
\hline \multirow{2}{*}{ Stage 8} & $\{1,2\}$ & $\{3\}$ & $\{4,5,6\}$ & $\{7\}$ \\
& $1 / 2$ & 2 & $13 / 3$ & 27 \\
\hline
\end{tabular}

Table 4: The fourth iteration of loop 3 in Example 6. The algorithm stops at Stage 8 with the final decision $((\{1,2\}, 1 / 2),(\{3\}, 2),(\{4,5,6\}, 13 / 3),(\{7\}, 27))$.

consistent social choice function.

Theorem 4. If $f(\cdot)$ is a single location function satisfying efficiency and participation and $\psi(\cdot)$ is a selection function of the correspondence $L A^{f}(\cdot)$, then any social choice function that maps each problem $(R, \bar{k}) \in \mathscr{D}$ into a decision obtained by the leftward adjustment of $\psi(R, \bar{k})$ satisfies both efficiency and consistency.

Proof. The result follows immediately from the definition of the leftward adjustment algorithm and Propositions 1 and 2.

Note that the leftward adjustment algorithm treats agents "symmetrically". Thus using $f(\cdot)$ and $\psi(\cdot)$ with the same symmetric treatment property, we can construct a large family of social choice functions satisfying "anonymity" as well as efficiency and consistency.

Proposition 2 and Theorem 4 improve Proposition 1 in Barberà and Beviá (2002) by showing existence of a larger family of self-selection consistent functions that can be obtained through the leftward adjustment algorithm. We show that any selection function $\psi(\cdot)$ of the correspondence $L A^{f}(\cdot)$ can be used in the algorithm to obtain a self-selection consistent function, while Barberà and Beviá (2002) use one special $\psi(\cdot)$ (decisions made by this function are explained in Footnote 5). Also by virtue of our conditions for efficiency, we are able to give a formal proof that the leftward adjustment algorithm leads to an efficient decision. The proof of Proposition 1 in Barberà and Beviá (2002) only shows that their construction leads to an outcome that satisfies local efficiency and no-envy, which are necessary but not sufficient for efficiency. To give more explanation in this regard, let us return to Example 6 and explain how we modify their construction.

In what follows, we use the same notation as used in the proof of Proposition 1 in Barberà and Beviá (2002). Their construction applied to Example 6 starts with the initial decision given by $C_{1}^{1}=\{1\}, x_{1}^{1}=0, C_{2}^{1}=\{2\}, x_{2}^{1}=1, C_{3}^{1}=\{3\}, x_{3}^{1}=2$, and $C_{4}^{1}=$ $\{4,5,6,7\}, x_{4}^{1}=10$. Since agent 6 in the fourth group prefers $x_{3}^{1}=2$ to $x_{4}^{1}=10$, there is an envy and $C_{3}=C_{3}^{1} \cup\{5,6\}$ (see p.272 of Barberà and Beviá 2002; in this example, 
$k=4$ and $\left.C_{k-1}=C_{3}\right)$. Note that $f\left(R_{C_{3}}\right)=11 / 3$ and there is no agent in $C_{4}$ whose peak is between $x_{3}^{1}=2$ and $f\left(R_{C_{3}}\right)=11 / 3$. Thus the process in lines 2-17 of p.272 is not needed. So $C_{3}^{2}=C_{3}=C_{3}^{1} \cup\{5,6\}=\{3,5,6\}, x_{3}^{2}=11 / 3, C_{4}^{2}=\{4,7\}, x_{4}^{2}=31 / 2$. And $C_{1}^{2}=\{1\}, x_{1}^{2}=0, C_{2}^{2}=\{2\}$, and $x_{2}^{2}=1$. Then there is no-envy and the process stops. However, the outcome $((\{1\}, 0),(\{2\}, 1),(\{3,5,6\}, 11 / 3),(\{4,7\}, 31 / 2))$ is not efficient because moving agent 3 from community 3 to community 2 (note that $1 I_{3} 11 / 3$ ) and changing the location for community 3 from $11 / 3$ to 4 makes both agents 5 and 6 better off without making anyone else worse off.

Therefore, there needs to be some change in their construction. The first change we can think of is the following: instead of checking no-envy as the stopping criterion, we check what is in Stage 2 in our algorithm. Unfortunately, this does not resolve the deficiency fully. To see this, let us continue with the above example. Since there is an agent, agent 3, who feels indifferent between his location and the left location (and agent 3 is the only such agent), we reiterate the whole process as in Barberà and Beviá (2002). Now we have to set $k$, at the top of p.272 in their paper, to be equal to 3. Then we obtain $C_{1}^{3}=\{1\}, x_{1}^{3}=0, C_{2}^{3}=C_{2}^{2} \cup\{3\}=\{2,3\}, x_{2}^{3}=3 / 2, C_{3}^{3}=\{5,6\}$, $x_{3}^{3}=9 / 2, C_{4}^{3}=\{4,7\}$, and $x_{4}^{3}=31 / 2$. Again check the criterion in our Stage 2. The last outcome does not pass the criterion and in fact in this case there is an envy (agent 4 prefers the location in community 3 to his own). So the next iteration starts. It yields $C_{1}^{4}=\{1\}, x_{1}^{4}=0, C_{2}^{4}=\{2,3\}, x_{2}^{4}=3 / 2, C_{3}^{4}=\{4,5,6\}, x_{3}^{4}=13 / 3, C_{4}^{4}=\{7\}$, and $x_{4}^{4}=27$. But note that $x_{3}^{4}<x_{3}^{3}$, in contradiction to the last sentence of the proof in Barberà and Beviá (2002), that is, ' $x_{h}^{j} \geq x_{h}^{j-1}$ for all $h \in\{1, \ldots, k\} \ldots$.'. Thus there needs to be some further change.

The change we made in this regard is that in our algorithm, we combine what might have been two or more iterations in Barberà and Beviá (2002) in one iteration of loop 3 (loop 1 and loop 2 are within loop 3). This guarantees monotonic movement of locations in every iteration as shown in Step 1 of the proof of Proposition 2 (so it resolves the problem mentioned in the previous paragraph).

Our Proposition 2 shows, in addition, that the algorithm can be used with any initial allocation satisfying the three properties, no-overlap, no-right-envy, and right stability. This gives more power to the result.

\section{A Proof of Proposition 2}

In this section, we prove Proposition 2.

The following two lemmas in Barberà and Beviá (2002) are useful.

Lemma 5 (Barberà and Beviá 2002). Consider a single location function $f$ satisfying 
participation and efficiency. Let $S, S^{\prime} \in \mathscr{N}$ be two non-empty disjoint sets of agents. Let $R \in \mathscr{R}^{S \cup S^{\prime}}$ and $y \in \mathbb{R}$. If for all $i \in S, p\left(R_{i}\right)<y$, and for all $i \in S^{\prime}, f\left(R_{S}\right) \leq p\left(R_{i}\right)<y$ and $f\left(R_{S}\right) R_{i} y$, then for all $i \in S^{\prime}$,

$$
f\left(R_{S}, R_{S^{\prime}}\right) R_{i} y \text { and } f\left(R_{S}\right) \leq f\left(R_{S}, R_{S^{\prime}}\right)<y .
$$

Lemma 6 (Barberà and Beviá 2002). Consider a single location function $f$ satisfying participation and efficiency. Let $S \in \mathscr{N}$ be a non-empty set of agents. Let $y \in \mathbb{R}$ be such that $y<f\left(R_{S}\right)$. If we let $S^{\prime} \equiv\left\{i \in S: y R_{i} f\left(R_{S}\right)\right\}$,

$$
f\left(R_{S}\right) \leq f\left(R_{S \backslash S^{\prime}}\right)
$$

Now we are ready to prove the proposition.

Proof of Proposition 2. Let $N \in \mathscr{N}, R \in \mathscr{R}^{N}$, and $\bar{k} \in \mathbb{N}$. To simply our notation, for each $S \subseteq N$, let $f(S)$ mean $f\left(R_{S}\right)$. Similarly let $p_{*}(S) \equiv p_{*}\left(R_{S}\right), p^{*}(S) \equiv p^{*}\left(R_{S}\right)$, and $p_{i} \equiv p\left(R_{i}\right)$. Let $z \equiv\left(C_{k}, x_{k}\right)_{k=1}^{\bar{k}}$ be an $N / \bar{k}$-decision satisfying the stated assumptions. Let $l$ be the maximum community index in Stage 1 . The proof is trivial if $l=1$. Suppose that $l>1$. Then $C_{l}^{*} \equiv\left\{i \in C_{l}: x_{l-1} R_{i} x_{l}\right\} \neq \emptyset$. The proof is in five steps.

Step 1. For each $k=1, \ldots, \bar{k}, x_{k} \leq x_{k}^{\prime}$ and if $k<\bar{k}, x_{k}^{\prime}<x_{k+1}$.

When $k \leq l-2$, the proof is trivial since $z^{\prime}$ is identical to $z$ for these communities and $z$ satisfies no-overlap.

Substep 1.1. $x_{l-1} \leq x_{l-1}^{\prime}<x_{l}$.

For each $i \in C_{l}^{*}$, since $x_{l-1} R_{i} x_{l}$ and $z$ satisfies no-overlap, then $x_{l-1}<p_{i}<x_{l}$. By Lemma 5,

$$
x_{l-1} \leq f\left(C_{l-1} \cup C_{l}^{*}\right)<x_{l} .
$$

Let $\hat{C}_{l-1} \equiv C_{l-1} \cup C_{l}^{*}$. Let $i \in C_{l} \backslash \hat{C}_{l-1}$ be the agent who moves into community- $(l-1)$ in the first iteration of loop 1 . Then $p_{i} \leq f\left(\hat{C}_{l-1}\right)$. By no-overlap at $z, x_{l-1}<p_{i}$. Thus from (6), we get

$$
x_{l-1}<p_{i} \leq f\left(\hat{C}_{l-1}\right)<x_{l}
$$

Clearly, $f\left(\hat{C}_{l-1}\right) P_{i} x_{l}$. Since $i \notin C_{l}^{*}, x_{l} P_{i} x_{l-1}$ and so $f\left(\hat{C}_{l-1}\right) P_{i} x_{l-1}$. By participation, $f\left(\hat{C}_{l-1} \cup\{i\}\right) R_{i} f\left(\hat{C}_{l-1}\right)$. Thus, $f\left(\hat{C}_{l-1} \cup\{i\}\right) P_{i} x_{l}$ and $f\left(\hat{C}_{l-1} \cup\{i\}\right) P_{i} x_{l-1}$. Therefore $f\left(\hat{C}_{l-1} \cup\{i\}\right)$ is closer to $i$ 's peak location $p_{i}$ than $x_{l}$ or $x_{l-1}$, which together with (7) implies

$$
x_{l-1}<f\left(\hat{C}_{l-1} \cup\{i\}\right)<x_{l} .
$$


The same argument applies for each iteration of loop 1 and at the end of loop 1, we get

$$
x_{l-1}<f\left(C_{l-1}^{\prime}\right)\left(=x_{l}^{\prime}\right)<x_{l}
$$

Note that $x_{l-1}<x_{l-1}^{\prime}$ when loop 1 is triggered.

Substep 1.2. For each $k \geq l, x_{k} \leq x_{k}^{\prime}$ and if $k<\bar{k}, x_{k}^{\prime}<x_{k+1}$.

We only prove the statement for $k=l$. The proof for $k>l$ is the same. In what follows, we assume $k<\bar{k}$ but our proof covers the case $k=\bar{k}$.

By no-overlap at $z$ and Lemma 6,

$$
x_{l-1}<x_{l}=f\left(C_{l}\right) \leq f\left(C_{l} \backslash C_{l}^{*}\right) .
$$

Let $i \in C_{l} \backslash C_{l}^{*}$ be the agent who moves into community- $(l-1)$ in the first iteration of loop 1. Then

$$
p_{i} \leq f\left(C_{l-1} \cup C_{l}^{*}\right)<x_{l} \leq f\left(C_{l} \backslash C_{l}^{*}\right),
$$

where the second inequality follows from (6) and the third follows from (10). By Lemma 6 , after moving $i$ into community- $(l-1)$ from community- $l$, the location for community-l moves to the right or stays constant, that is, $f\left(C_{l} \backslash C_{l}^{*}\right) \leq f\left(\left(C_{l} \backslash C_{l}^{*}\right) \backslash\{i\}\right)$. Applying the same argument for each iteration of loop 1 with $k=l-1$ (moving agents from community- $l$ to community- $(l-1))$, we obtain $f\left(C_{l} \backslash C_{l}^{*}\right) \leq f\left(\hat{C}_{l}\right)$, where $\hat{C}_{l}=$ $C_{l} \backslash C_{l-1}^{\prime}$. Then from (11),

$$
x_{l} \leq f\left(C_{l} \backslash C_{l}^{*}\right) \leq f\left(\hat{C}_{l}\right) .
$$

Since $f\left(\hat{C}_{l}\right) \leq p^{*}\left(\hat{C}_{l}\right) \leq p^{*}\left(C_{l}\right)<x_{l+1}$ (the last inequality holds by no-overlap at $z$ ),

$$
x_{l} \leq f\left(C_{l} \backslash C_{l}^{*}\right) \leq f\left(\hat{C}_{l}\right)<x_{l+1} .
$$

We now consider loop 1 with $k=l$ (moving agents from community- $(l+1)$ to community-l). Let $i \in C_{l+1}$ be the agent who moves into community-l in the first round of loop 1 . Then $p_{i} \leq f\left(\hat{C}_{l}\right)$ and by no-overlap at $z, x_{l}<p_{i}$. Thus $x_{l}<p_{i} \leq f\left(\hat{C}_{l}\right)<x_{l+1}$. By participation, $f\left(\hat{C}_{l} \cup\{i\}\right) R_{i} f\left(\hat{C}_{l}\right)$. By definition of $l$ in Stage $1, x_{l+1} P_{i} x_{l}$. Then $f\left(\hat{C}_{l}\right) P_{i} x_{l+1} P_{i} x_{l}$. Therefore $x_{l}<f\left(\hat{C}_{l} \cup\{i\}\right) \leq f\left(\hat{C}_{l}\right)<x_{l+1}$. Applying the same argument for each iteration of loop 1 , we finally get $x_{l}<f\left(C_{l}^{\prime}\right)=x_{l}^{\prime}<x_{l+1}$.

Step 2. Decision $z^{\prime}$ obtained after each iteration of loop 3 satisfies no-overlap.

We show that $x_{1}^{\prime}<p_{*}\left(C_{2}^{\prime}\right), p^{*}\left(C_{\bar{k}-1}^{\prime}\right)<x_{\bar{k}}^{\prime}$, and for each $k \in\{2, \ldots, \bar{k}-1\}, p^{*}\left(C_{k-1}^{\prime}\right)<$ $x_{k}^{\prime}<p_{*}\left(C_{k+1}^{\prime}\right)$. For $k<l-1$, the statement follows directly from no-overlap at $z$ because loop 3 does not change anything for these communities.

Substep 2.1. For each $k \in\{l-1, \ldots, \bar{k}-1\}, x_{k}^{\prime}<p_{*}\left(C_{k+1}^{\prime}\right)$. 
We only consider the case $k=l-1$ and skip the same arguments for other cases. For each $i \in \hat{C}_{l} \equiv C_{l} \backslash C_{l-1}^{\prime}, x_{l-1}^{\prime}<p_{i}$ because otherwise loop 1 for $k=l-1$ should not have ended at $C_{l-1}^{\prime}$ and $x_{l-1}^{\prime}$. Hence

$$
x_{l-1}^{\prime}<p_{*}\left(\hat{C}_{l}\right)
$$

Let $i \in C_{l+1}$ be the agent who moves into community-l in the first iteration of loop 1 . If $p_{i} \leq x_{l-1}^{\prime}$, then $p_{i} \leq x_{l-1}^{\prime}<x_{l}$ by Step 1 and so $i \in C_{l+1}$ prefers $x_{l}$ to $x_{l+1}$, contradicting definition of $l$ in Stage 1. Hence $x_{l-1}^{\prime}<p_{i}$. Combining this with (14), $x_{l-1}^{\prime}<p_{*}\left(\hat{C}_{l} \cup\right.$ $\{i\})$. Applying the same argument for each iteration of loop 1, we show that $x_{l-1}^{\prime}<$ $p_{*}\left(C_{l}^{\prime}\right)$.

Substep 2.2. For each $k \in\{l-2, \ldots, \bar{k}-1\}, p^{*}\left(C_{k}^{\prime}\right)<x_{k+1}^{\prime}$.

We only consider the case $k=l-1$ and skip the same arguments for other cases. Note that each iteration of loop 1 for each $k$ does not change the maximal peak for community- $k$. Thus $p^{*}\left(C_{l-1}^{\prime}\right)=p^{*}\left(C_{l-1} \cup C_{l}^{*}\right)$. By no-overlap at $z$ and the definition of $C_{l}^{*}, p^{*}\left(C_{l-1} \cup C_{l}^{*}\right)<x_{l}$. By Step $1, x_{l} \leq x_{l}^{\prime}$. Therefore, $p^{*}\left(C_{l-1}^{\prime}\right)<x_{l}^{\prime}$.

Step 3. For each $k \geq 2$ and each $i \in C_{k-1}^{\prime}, x_{k-1}^{\prime} R_{i} x_{k}^{\prime}$.

For $k \leq l-1$, the result follows directly from the condition of no-right-envy at $z$ stated in (4). We consider below only the case $k=l$. The same argument can be used for each $k \geq l+1$. Let $i \in C_{l-1}^{\prime}$ be such that $p_{i} \leq x_{l-1}^{\prime}$. Since $x_{l-1}^{\prime}<x_{l}^{\prime}, x_{l-1}^{\prime} P_{i} x_{l}^{\prime}$. Let $i \in C_{l-1}^{\prime}$ be such that $p_{i}>x_{l-1}^{\prime}$. If $i \in C_{l-1}^{\prime} \cap C_{l-1}$, then by (4), $x_{l-1} R_{i} x_{l}$. By nooverlap at $z, p_{i}<x_{l}$. Since $x_{l-1} \leq x_{l-1}^{\prime}$ and $x_{l} \leq x_{l}^{\prime}$, as shown in Step 1, and $x_{l-1}^{\prime}<x_{l}^{\prime}$, then $x_{l-1}^{\prime} R_{i} x_{l}^{\prime}$. If $i \in C_{l-1}^{\prime} \backslash C_{l-1}, i \in C_{l}^{*}$ or $i \notin C_{l}^{*}$. In the former case, $x_{l-1} R_{i} x_{l}$ and so using the same argument as above, we show $x_{l-1}^{\prime} R_{i} x_{l}^{\prime}$. In the latter case, suppose that $i$ is the person who moves into community- $(l-1)$ in the first iteration of loop 1. Then $f\left(C_{l-1} \cup C_{l}^{*}\right)$ is the location right before $i$ moves. And if we let $y_{h} \equiv$ $\min \left\{y: y I_{h} f\left(C_{l-1} \cup C_{l}^{*}\right)\right\}$ for all $h \in C_{l}$ with $p_{h} \leq f\left(C_{l-1} \cup C_{l}^{*}\right)$, then $y_{i} \leq y_{h}$ for all $h \in$ $C_{l}$ with $p_{h} \leq f\left(C_{l-1} \cup C_{l}^{*}\right)$ (see the condition in Stage 5.1.1). Since by participation, $i$ should be weakly better off after moving, the new location for community- $(l-1)$, denoted by $\hat{x}_{l-1}$, should be in $\left[y_{i}, f\left(C_{l-1} \cup C_{l}^{*}\right)\right]$. Note that for each $h \in C_{l}$, if $p_{h} \leq \hat{x}_{l-1}$, then $h$ is a person with $p_{h} \leq f\left(C_{l-1} \cup C_{l}^{*}\right)$ (recall that among these persons, $i$ has the lowest $y_{h}$ value), which implies $y_{i} \leq y_{h}$. Thus $y_{i} \leq p_{h}$ (otherwise, $y_{h} \leq p_{h}<y_{i}$, contradicting $\left.y_{i} \leq y_{h}\right)$. Let $h$ be the person who moves into community- $(l-1)$ in the second iteration of loop 1 . Then using the same argument as above we can show that the location after $h$ moves, should be in $\left[y_{h}, \hat{x}_{l-1}\right] \subseteq\left[y_{i}, f\left(C_{l-1} \cup C_{l}^{*}\right)\right]$. Similarly, after each iteration of loop 1 , we get a location for community- $(l-1)$ in $\left[y_{i}, f\left(C_{l-1} \cup C_{l}^{*}\right)\right]$. Hence $x_{l-1}^{\prime} \in\left[y_{i}, f\left(C_{l-1} \cup C_{l}^{*}\right)\right]$. Therefore $x_{l-1}^{\prime} R_{i} f\left(C_{l-1} \cup C_{l}^{*}\right) P_{i} x_{l} R_{i} x_{l}^{\prime}$, where 
the second and the third relations follow from $p_{i} \leq f\left(C_{l-1} \cup C_{l}^{*}\right)<x_{l} \leq x_{l}^{\prime}$. The same argument can be used for every other agent who moves into community- $(l-1)$ in loop 1.

Step 4. For each $k \leq \bar{k}-1$, there is $j \in C_{k}^{\prime}$ such that $x_{k}^{\prime} P_{j} x_{k+1}^{\prime}$ and $x_{k}^{\prime} \leq p_{j}$.

For $k \leq l-2$, this follows from the condition of right stability at $z$ stated in (5), and $x_{l-1} \leq x_{l-1}^{\prime}$ shown in Step 1. Let $k=l-1$. There are two cases, $x_{l-1}=x_{l-1}^{\prime}$ or $x_{l-1}<x_{l-1}^{\prime}$. In the former case, we simply use (5) and $x_{l} \leq x_{l}^{\prime}$ to show existence of $j \in C_{l-1}$ with the two desired properties. Consider the latter case $x_{l-1}<x_{l-1}^{\prime}$. If there is $j \in C_{l-1}$ with $p_{j} \geq x_{l-1}^{\prime}$, this is a person we are looking for because by (4) and $x_{l-1}<x_{l-1}^{\prime}$, we have $x_{l-1}^{\prime} P_{j} x_{l-1} R_{j} x_{l} R_{j} x_{l}^{\prime}$. Suppose that there is no $j \in C_{l-1}$ with $p_{j} \geq x_{l-1}^{\prime}$. Then by efficiency of $f$, there is $j \in C_{l}^{*}$ with $p_{j} \geq x_{l-1}^{\prime}$. And since $x_{l-1}<x_{l-1}^{\prime}$, then by definition of $C_{l}^{*}$ (note $x_{l-1} R_{j} x_{l}$ ), $x_{l-1}^{\prime} P_{j} x_{l-1} R_{j} x_{l} R_{j} x_{l}^{\prime}$.

Now consider $k=l$. If $x_{l}=x_{l}^{\prime}$, the result follows immediately from the condition of right stability at $z$ in (5), and $x_{l+1} \leq x_{l+1}^{\prime}$ as shown in Step 1. Suppose $x_{l}<x_{l}^{\prime}$. Since $p^{*}\left(C_{l}\right)=p^{*}\left(C_{l}^{\prime}\right)$, then for each $j \in C_{l} \cap C_{l}^{\prime}$ with $p_{j}=p^{*}\left(C_{l}^{\prime}\right)=p^{*}\left(C_{l}\right)$, we have $x_{l}<x_{l}^{\prime} \leq p_{j}<x_{l+1} \leq x_{l+1}^{\prime}$ and so $x_{l}^{\prime} P_{j} x_{l} R_{j} x_{l+1} R_{j} x_{l+1}^{\prime}$, where the second relation holds by (4). The same argument applies for all other $k \geq l$.

Step 5. We now complete our proof. Each iteration of loop 3 moves at least one agent (in fact all agents in $C_{l}^{*}$ ) from one community into the left adjacent community. Since there is only a finite number of agents, this process must end after a finite number of iterations. And after the last iteration, for each $k \in\{2, \cdots, \bar{k}\}, C_{k}^{*}=\emptyset$, which means that for each $k \in\{2, \cdots, \bar{k}\}$ and each $i \in C_{k}, x_{k} P_{i} x_{k-1}$. This property together with (4) and (5) shown by Steps 1-4 imply that $z^{\prime}$ passes the neighbor test. Therefore, by Theorem 2, $z^{\prime}$ satisfies efficiency.

\section{References}

[1] Aumann, R.J. (1959), "Acceptable points in general cooperative n-person games", in Contributions to the Theory of Games, Vol. IV, Princeton Univ. Press, Princeton.

[2] Barberà, S. and C. Beviá (2002), "Self-selection consistent functions", Journal of Economic Theory 105(2):263-277.

[3] Barberà, S. and C. Beviá (2005), "Locating public facilities by majority: Stability, consistency and group formation", Games and Economic Behavior, forthcoming.

[4] Ehlers, L. (2002), "Multiple public goods and lexicographic preferences: replacement principle", Journal of Mathematical Economics 37:1-15. 
[5] Ehlers, L. (2003), "Multiple public goods, lexicographic preferences, and singleplateaued preference rules", Games and Economic Behavior 43:1-27.

[6] Konishi, H., M. Le Breton, and S. Weber (1997a), "Pure strategy Nash equilibrium in a group formation game with positive externalities", Games and Economic Behavior 21:161-182.

[7] Konishi, H., M. Le Breton, and S. Weber (1997b), "Equilibria in a model with partial rivalry", Journal of Economic Theory 72:225-237.

[8] Konishi, H., M. Le Breton, and S. Weber (1999), "On coalition-proof Nash equilibria in common agency games", Journal of Economic Theory 85:122-139.

[9] Miyagawa, E. (2001), "Locating libraries on a street", Social Choice and Welfare 18(3):527-541.

[10] Moulin, H. (1994), "Serial cost-sharing of excludable public goods", Review of Economic Studies 61:305 325. 